\title{
ANÁLISIS DE DOS TIPOS DE REFUERZO A FLEXIÓN DE FORJADOS DE SEMIVIGUETAS
}

\author{
(ANALYSIS OF KINDS OF FLEXURAL STRENGTHENING OF JOIST CONSTRUCTION \\ SLABS)
}

José L. Ramírez, Dr. Ing. Industrial

José M. Bárcena, Dr. Ing. Industrial

Pedro J. Landa, Ing. Industrial

Fecha de recepción: 4-XI-88

RESUMEN

Los forjados constituyen el elemento estructural de mayor incidencia económica en el coste de un edificio de plantas, alcanzando porcentajes respecto al valor total del inmueble que superan habitualmente el $20 \%$.

Por ello, y continuando trabajos anteriores en torno a pilares y vigas, se está desarrollando por los autores un proyecto de investigación relativo al refuerzo de forjados, tanto en forma de losa maciza como confeccionados a partir de semiviguetas $\left({ }^{*}\right)$.

En el presente artículo se exponen los resultados del refuerzo a flexión adhiriendo malla de acero a la parte inferior de los forjados de semiviguetas por medio de mortero adherente u hormigón proyectado. Se comprueban muy buenos resultados, difícilmente esperables contando con que la nueva armadura está adherida en un $85 \%$ a la parte inferior de las bovedillas de relleno, sin consideración resistente en el cálculo, y sólo en un $15 \%$ a la semivigueta resistente.
SUMMARY

Slabs are the most costly structural elements in a building, often accounting for more than $20 \%$ of the cost of the building.

Therefore, and as a continuation of previous works on columns and beams, the authors are working on a research project relative to the strengthening of slabs, both massive concrete slabs and those in joist construction form.

This article presents the results of flexural strengthening carried out by attaching steel mesh to the bottom of joist construction slabs with adhesive mortar or shot concrete. Very good results were obtained, which are hard to explain, if it is taken into account that $85 \%$ of the reinforcement is adhered to the porous concrete filling piedes between ribes which are of no significant strength in the calculation, and only $15 \%$ to the resistant ribs bottoms.

\section{INTRODUCCIÓN}

Con motivo del programa de investigación que vienen realizando los autores sobre refuerzos de forjados de hormigón, se presentan en este artículo los resultados más relevantes del desarrollo de una parte del programa llevada a cabo sobre forjados de semiviguetas. Se trataba de añadir capacidad flectora en la zona de momentos positivos adhiriendo armadura en la parte inferior del forjado, y ello de dos modos diferentes.

\section{La necesidad de reforzar los forjados puede provenir}

(*) Trabajo realizado en la Escuela Superior de Ingenieros Industriales de Bilbao y en LABEIN con subvención CAICYT. Constituye parte de la experimentación de los trabajos de tesis doctoral de P. J. Landa. de diferentes circunstancias, como errores de proyecto, deficiencias de ejecución o reparación de daños, pero, en nuestra experiencia, la mayor parte de las veces viene exigida por la ampliación de las cargas de uso de alguna zona del edificio.

El forjado inicial de partida ha sido proyectado para las cargas usuales en viviendas sencillas y las luces habituales de alrededor de $5 \mathrm{~m}$, con continuidad entre los diversos vanos. Esta continuidad ha permitido ensayar paños de forjado de $4 \mathrm{~m}$ de luz, simplemente apoyados en los extremos de la luz, estáticamente equivalentes a los reales respecto al refuerzo de las zonas de momentos positivos. No se ha tratado en este trabajo del refuerzo de las zonas de apoyo o de momento negativo. 
Las pruebas se han efectuado sobre nueve elementos, preparados con el siguiente criterio:

- Tres forjados se dejaron sin reforzar, como referencia para comparaciones posteriores.

- Tres forjados se reforzaron mediante añadido de una malla inferior con recubrimiento de la misma mediante mortero adherente.

- Tres forjados se reforzaron mediante añadido de una malla inferior recubierta mediante hormigón proyectado.

En la bibliografía (2), (5), se citan métodos de reparación a base de recrecido de la capa compresora, intercalado de viguetas adicionales, pegado de chapas o añadido de armaduras. Sin embargo, el refuerzo, chapa delgada o armadura, se suele aplicar por la parte inferior directamente sobre el elemento estructural resistente, de tal forma que la transmisión de esfuerzos se concentra en una zona estructuralmente fiable. Nuestro propósito, contrariamente, ha sido considerar una distribución uniforme del mallazo en la parte inferior del forjado indiscriminadamente entre la zapatilla de la semivigueta y la bovedilla de hormigón poroso.

Ello facilita la ejecución y proporciona monolitismo relativamente a las soluciones tradicionales pero, sin embargo, deja como muy cuestionable y difícil de justificar por cálculo la colaboración de la parte del refuerzo que queda adherida a las bovedillas, piezas sin misión estructural.

\section{DIMENSIONAMIENTO DE LOS FORJADOS Y DE SUS REFUERZOS}

El sistema de partida ha sido un forjado semirresistente de hormigón armado del tipo que se muestra en la figura 1.
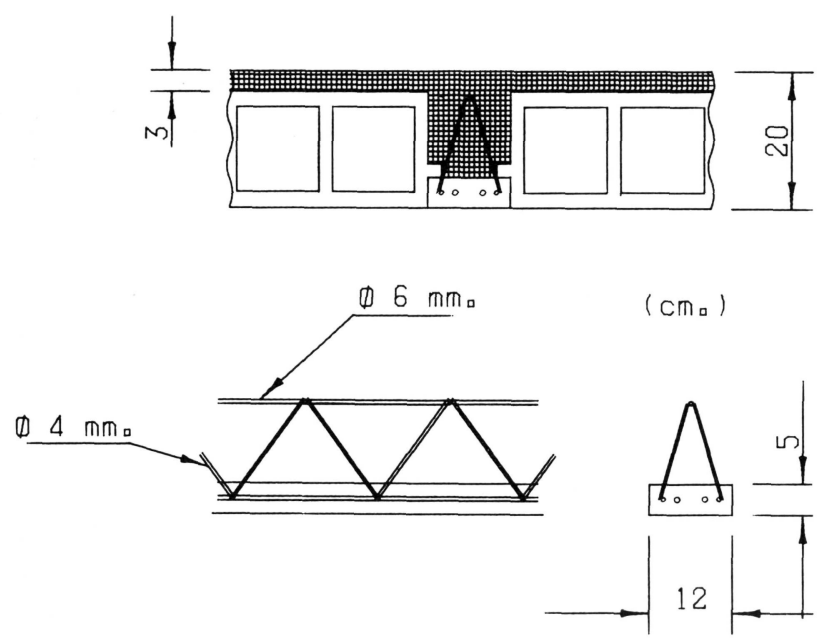

Fig. -1

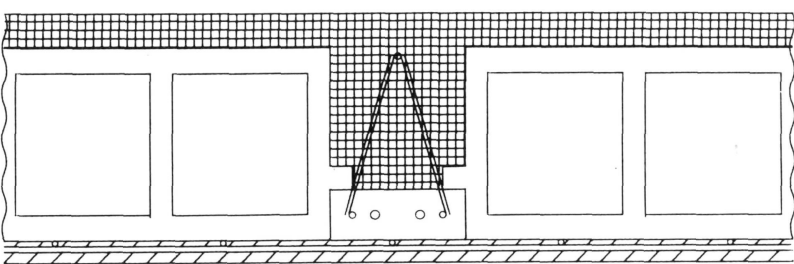

Fig. -2

A nivel de especificación se compone de una zapatilla de hormigón de alta calidad (fck $=250 \mathrm{kp} / \mathrm{cm}^{2}$ ), y de dos celosías conformadas entre tres redondos de acero de alta adherencia $\left(\mathrm{fyk}=5.100 \mathrm{kp} / \mathrm{cm}^{2}\right)$. Las celosías, de diagonales consecutivas en ambos sentidos, son de acero de análoga calidad. Las bovedillas son de hormigón poroso, y el nervio y la capa compresora son de hormigón colado in situ de resistencia teórica $\mathrm{fck}=175 \mathrm{kp} / \mathrm{cm}^{2}$.

El planteamiento de los ensayos sigue el siguiente esquema. Se ha elegido un forjado de partida que resis. te teóricamente una determinada sobrecarga actuante sobre el mismo. En función de unas supuestas necesidades de mayor sobrecarga se han diseñado los correspondientes forjados reforzados, cuya carga de rotura teórica se ha calculado por las formulaciones usuales de instrucciones y códigos. La relación entre los valores teóricos de agotamiento entre uno y otro caso nos da el nivel de refuerzo proyectado. La comparación entre dichos valores y los resultados obtenidos en los ensayos nos da el grado de adecuación de las teorías empleadas en el dimensionamiento de los forjados.

Los refuerzos han consistido en $7 \varnothing 5$ por metro en la dirección de las viguetas más una armadura transversal de reparto, resultando la sección indicada en la fi. gura 2, siendo todo el acero de alta resistencia. La forma de ejecución de los refuerzos se explica en el apartado siguiente.

El cálculo a flexión del forjado inicial se ha efectuado de acuerdo con el método de los estados límites y diagrama parábola rectángulo, encontrándose la línea neutra en la capa compresora.

Para los forjados reforzados se ha supuesto la armadura total (compuesta por la de la zapatilla prefabricada más la adherida correspondiente del refuerzo) concentrada en el centro de gravedad e idénticas hipótesis de cálculo.

El forjado de partida ha sido el de la figura 1 con una armadura por vigueta de $2 \varnothing 6$ y $2 \varnothing 8$ lo cual corresponde a un momento de cálculo de catálogo por vigueta (usando los coeficientes de minoración habituales $\gamma_{\mathrm{s}}=1,15$ y $\left.\gamma_{\mathrm{c}}=1,5\right)$ próximo a $1.200 \mathrm{~m} \cdot \mathrm{kg}$, siendo el intereje de $70 \mathrm{~cm}$. 
CUADRO N. 1

\begin{tabular}{|c|c|c|c|c|c|c|c|c|c|c|}
\hline \multirow{2}{*}{ MATERIALES } & \multicolumn{10}{|c|}{$\begin{array}{l}\text { RESISTENCIAS (kp/cm²) } \\
\text { FORJADO NÚMERO }\end{array}$} \\
\hline & 1 & 2 & 3 & 4 & 5 & 6 & 7 & 8 & 9 & MEDIA \\
\hline $\begin{array}{l}\text { HORMIGÓN } \\
\text { INICIAL (28d) }\end{array}$ & $\begin{array}{l}164.1 \\
169.2\end{array}$ & $\begin{array}{l}161.3 \\
146.0\end{array}$ & $\begin{array}{l}136.4 \\
164.7\end{array}$ & $\begin{array}{l}148.8 \\
152.2\end{array}$ & $\begin{array}{l}149.9 \\
156.2\end{array}$ & $\begin{array}{l}142.6 \\
136.9\end{array}$ & $\begin{array}{l}131.3 \\
138.1\end{array}$ & $\begin{array}{l}139.2 \\
143.7\end{array}$ & $\begin{array}{l}149.9 \\
154.5\end{array}$ & $\begin{array}{c}\text { fcm } \\
149.1\end{array}$ \\
\hline $\begin{array}{l}\text { HORMIGÓN } \\
\text { INICIAL } \\
117 \text { dias } \\
\text { (edad rotura) }\end{array}$ & $\begin{array}{l}205.4 \\
203.7 \\
181.6 \\
174.3\end{array}$ & $\begin{array}{l}174.8 \\
196.9 \\
187.3 \\
189.0\end{array}$ & $\begin{array}{l}165.8 \\
174.2 \\
203.1 \\
199.2\end{array}$ & $\begin{array}{l}182.2 \\
189.0 \\
202.6 \\
198.0\end{array}$ & $\begin{array}{l}190.1 \\
181.6 \\
199.2 \\
194.3\end{array}$ & $\begin{array}{l}175.4 \\
182.8 \\
168.6 \\
164.6\end{array}$ & $\begin{array}{l}160.7 \\
143.1 \\
152.2 \\
170.9\end{array}$ & $\begin{array}{l}192.9 \\
190.1 \\
174.3 \\
175.1\end{array}$ & $\begin{array}{l}175.4 \\
174.8 \\
198.6 \\
194.6\end{array}$ & $\begin{array}{l}f c m \\
182.9\end{array}$ \\
\hline \multirow{2}{*}{$\begin{array}{l}\text { HORMIGÓN DEL } \\
\text { GUNITADO }\end{array}$} & \multicolumn{2}{|c|}{28 dias } & $\begin{array}{l}193.7 \\
201.5 \\
214.0 \\
\end{array}$ & $\begin{array}{c}\mathrm{fcm} \\
203.0\end{array}$ & \multirow{2}{*}{\multicolumn{2}{|c|}{$\begin{array}{l}\text { HORMIGÓN } \\
\text { DE LAS } \\
\text { SEMIVIGUETAS } \\
\text { PREFABRICADAS }\end{array}$}} & \multicolumn{2}{|c|}{28 días } & $\begin{array}{l}274.4 \\
278.4 \\
\end{array}$ & $\begin{array}{c}f c m \\
276.4\end{array}$ \\
\hline & \multicolumn{2}{|c|}{$\begin{array}{l}46 \text { dias } \\
\text { (edad rotura) }\end{array}$} & $\begin{array}{l}221.5 \\
220.9 \\
211.5\end{array}$ & $\begin{array}{l}f c m \\
217.9\end{array}$ & & & \multicolumn{2}{|c|}{$\begin{array}{c}150 \text { días } \\
\text { (edad rotura) }\end{array}$} & $\begin{array}{l}327.6 \\
337.2 \\
341.2 \\
337.8\end{array}$ & $\begin{array}{c}f \mathrm{~cm} \\
335.9\end{array}$ \\
\hline \multicolumn{3}{|c|}{$\begin{array}{r}\text { ARMADURAS SEMIVIGUETAS } \varnothing 6 \\
\varnothing 8\end{array}$} & $\begin{array}{c}\text { fyk } \\
8276 \\
5869\end{array}$ & $\begin{array}{c}\text { fyr } \\
8452 \\
7410\end{array}$ & \multicolumn{4}{|c|}{ ARMADURA DE MALLAZO $\varnothing 5$} & $\begin{array}{l}\text { fyk } \\
6656\end{array}$ & $\begin{array}{c}\text { fyr } \\
6871\end{array}$ \\
\hline
\end{tabular}

Para los forjados reforzados se ha distribuido un mallazo por la parte inferior de calidad teórica fyk = $5.100 \mathrm{kp} / \mathrm{cm}^{2}$, uniformemente repartido a razón de $7 \varnothing 5$ por $\mathrm{m}$. Este mallazo unido a la armadura original de la vigueta da lugar a un momento teórico de cálculo del elemento reforzado (con los mismos criterios anteriores) de aproximadamente $1.900 \mathrm{~m} . \mathrm{kg}$ para un ancho de forjado de $70 \mathrm{~cm}$. El refuerzo corresponde pues a un aumento de resistencia en valores teóricos de cálculo del orden del $60 \%$.

Ello equivale a que se puede lograr elevar la carga de servicio del forjado de $150 \mathrm{~kg} / \mathrm{m}^{2}$ a $400 \mathrm{~kg} / \mathrm{m}^{2}$, habiendo tenido en cuenta el aumento del peso propio correspondiente al refuerzo.

Habria que asegurarse, antes de verificar la resistencia a rasante entre refuerzo y forjado original, que el forjado reforzado es capaz de resistir los otros efectos del mayor esfuerzo cortante presente al aumentarse la carga de servicio. En otro caso se necesitaría, igualmente, hacer los refuerzos correspondientes.

Los momentos de cálculo dados anteriormente corresponden a valores teóricos de los materiales, que no son los reales, por lo que, para poder compararlos con los resultados experimentales, habría que corregirlos en función de las características reales de dichos materiales. Un resumen de las mismas se dan en el cuadro n. 1 . Se ha comprobado la consideración de no resis- tente de las bovedillas desde el punto de vista de cálculo $\left(\mathrm{fck}=140 \mathrm{kp} / \mathrm{cm}^{2}<175\right)$.

Rehechos todos los cálculos con las características reales de los materiales, manteniendo las hipótesis y métodos de cálculo anteriormente descritos y utilizando un coeficiente de cansancio del hormigón de 0,95, puesto que los ensayos se realizan en un tiempo breve, se obtienen para los diferentes forjados los siguientes valores medios de rotura teóricos:

\begin{tabular}{|c|c|}
\hline & $\begin{array}{l}\text { Momento por } \\
\text { vigueta } \\
\text { centro vano } \\
\text { (m.kp) }\end{array}$ \\
\hline $\begin{array}{l}\text { ginal . } \\
\text { fuerzo armadura inferior adherida }\end{array}$ & 1.830 \\
\hline morzo armadura inferior y hormigón & 3.031 \\
\hline oyectado & 3.031 \\
\hline
\end{tabular}

\section{PROCESO OPERATIVO DE LOS REFUERZOS}

Los refuerzos se han efectuado a partir de forjado descargado incluso de gran parte del peso propio merced a un apuntalamiento activo. 


\section{Refuerzo con armadura inferior y mortero adherente}

Ha consistido en las siguientes operaciones:

a) Colocación de una malla por la parte inferior, colgándola de los huecos de las bovedillas mediante anclajes adecuados de simple suspensión.

b) Puesta en contacto de la malla con la parte inferior del forjado por apriete de tuercas en el vástago de los anclajes.

c) No se cuidó especialmente la limpieza de la cara del forjado en contacto con las armaduras para simular las condiciones de obra.

d) Previamente a la aplicación del mortero se dio una capa de imprimación a forjado y armadura con un adhesivo del tipo látex.

e) Aplicación del mortero adherente mediante la llana por capas sucesivas hasta alcanzar el recubrimiento necesario de la malla (Foto n. ${ }^{\circ}$ 1).

El mortero empleado es uno de los recomendados para reparaciones de estructuras. Está modificado con polímeros y ofrece unas características del orden de 250 $\mathrm{kp} / \mathrm{cm}^{2}$ a compresión a 28 días para una relación agua/producto de 0,2 . El endurecimiento o fraguado es rápido y la resistencia a compresión es del orden de $100 \mathrm{kp} / \mathrm{cm}^{2}$ al primer día. Un inconveniente relativo del método es el coste del material, y la laboriosidad de colocación, puesto que hay que aplicar capas pequeñas para que no se descuelgue, y entre capas se debe de dar una nueva imprimación cuando haya varias horas de intervalo entre ellas. Lo anterior hace desaconsejable su uso para superficies importantes.

\section{Refuerzo con armadura inferior y hormigón proyectado}

La metodología utilizada en este tipo de refuerzo es análoga al caso anterior en los apartados a), b) y c):

d) Proyección del hormigón mediante máquina de gunitar por vía seca. Al comienzo de la proyección se utilizaba la manguera de la máquina para humedecer la zona proyectada (Foto $n^{\circ} 2$ ).

e) Corrección de los defectos en la zona inferior gunitada y posterior enlucido de la misma.

El hormigón proyectado ha sido de tamaño máximo de árido de $8 \mathrm{~mm}$ y una resistencia a 28 días de $200 \mathrm{kp} / \mathrm{cm}^{2}$. Las dificultades de ejecución son debidas a los rebotes del material, a la imprecisión de la proyección, y a la necesidad de maquinaria especial, lo que hace poco adecuado el procedimiento para reparación de superficies pequeñas y obras terminadas.

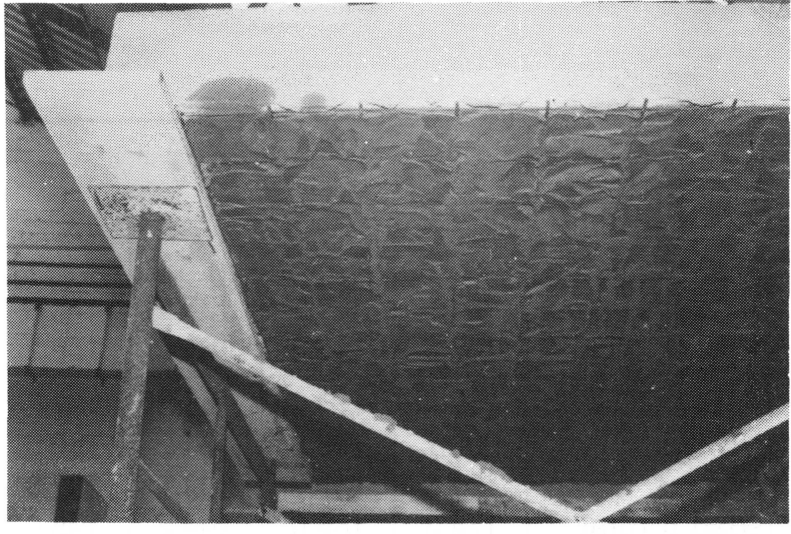

Foto 1

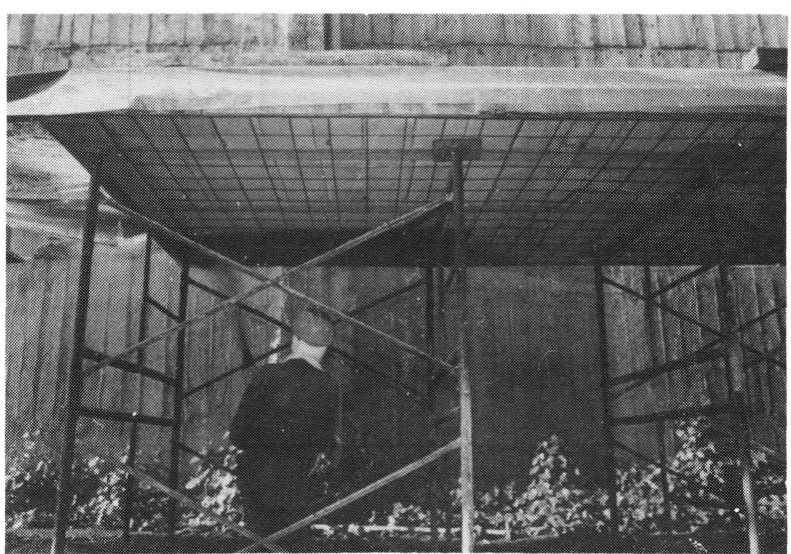

Foto 2

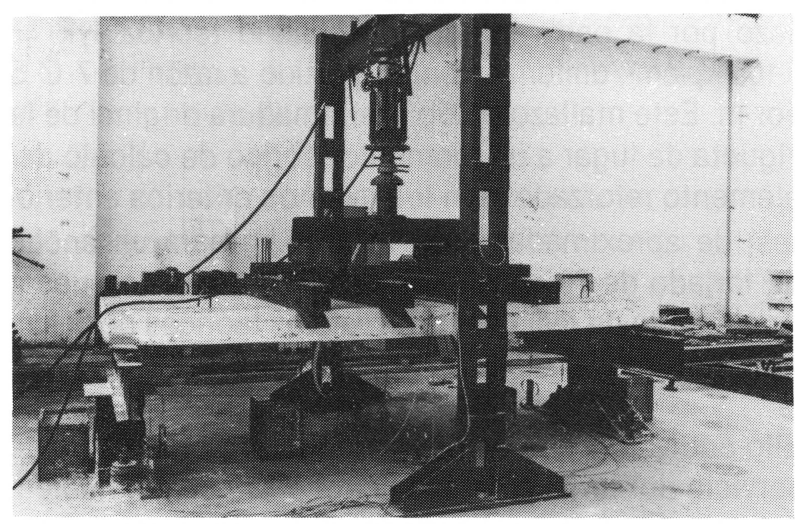

Foto 3

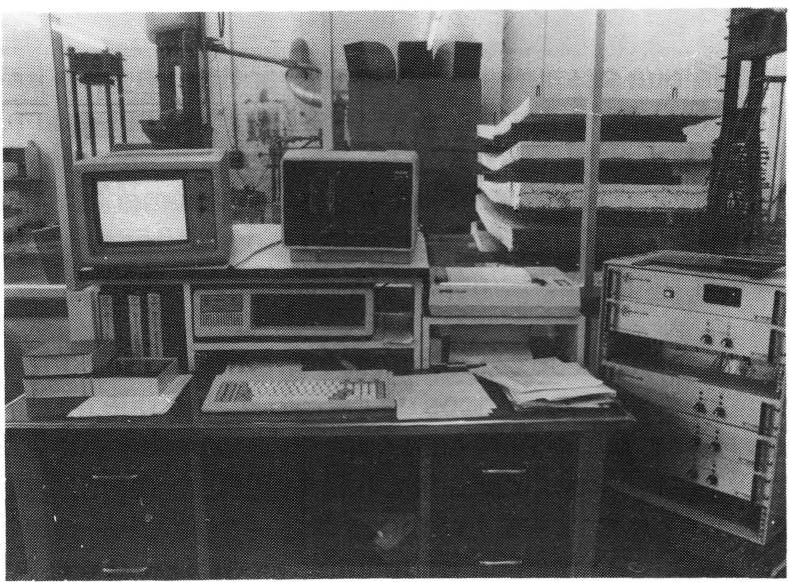

Foto 4

http://informesdelaconstruccion.revistas.csic.es 


\section{DESARROLLO DE LOS ENSAYOS}

Los diferentes forjados de 2,1 m de anchura se ensayaron a flexión como simplemente apoyados con una luz de $4 \mathrm{~m}$. Las cargas se aplicaron sobre cuatro líneas transversales a la luz del forjado separadas $1 / 7$ de la luz, quedando las líneas exteriores a 2/7 de la luz de los apoyos respectivos. El motivo de utilizar esta distribución de cargas es la gran similitud entre el diagrama de momentos flectores correspondiente a este esquema de cargas con el que producen cargas uniformemente distribuidas. El esfuerzo cortante máximo en el apoyo es, sin embargo, el $75 \%$ del que correspondería al caso de carga uniformemente repartida e igual momento flector. (Foto $n .^{\circ} 3$ ).

El proceso de carga seguido en todos los casos fue:

- Aumento sucesivo de cargas por escalones hasta llegar al estado teórico de servicio de cada tipo de forjado.

- Descarga.

- Carga hasta la rotura por escalones sucesivos.
Simultáneamente al proceso de cargas se median las flechas producidas en los forjados y las microdeformaciones de las armaduras de las viguetas y de los refuerzos mediante galgas extensométricas pegadas a las mismas antes del hormigonado. (Foto n. ${ }^{\circ}$ ).

Asímismo en cada nivel de cargas se procedía a una inspección visual de la fisuración y otras incidencias reseñables.

En el cuadro n. ${ }^{\circ} 2$ se puede ver un resumen de los resultados más significativos de los ensayos efectuados, incluyendo los valores individuales de los 9 forjados y las medias por grupos. También se incluye el gráfico $\mathrm{n}^{\circ} 1$ con la relación momento-flecha, de los tres tipos de ensayo.

\section{MODOS DE ROTURA}

\section{Forjados sin reforzar}

En los tres elementos ensayados se produjo una rotura por flexión en el centro del vano, rompiendo las armaduras de $\varnothing 6$ de las viguetas.

CUADRO N. ${ }^{\circ} 2$

RESULTADOS SIGNIFICATIVOS DEL ENSAYO DE REFUERZOS EN FLEXION (Valores por vigueta)

\begin{tabular}{|c|c|c|c|c|c|c|}
\hline \multirow[t]{2}{*}{$\begin{array}{l}\text { Tipo } \\
\text { forjado }\end{array}$} & \multirow{2}{*}{$\begin{array}{c}\text { Momento } \\
\text { de } \\
\text { rotura } \\
\text { (m.kp) }\end{array}$} & \multicolumn{2}{|c|}{$\begin{array}{c}\text { Momento de fisuración } \\
\text { (m.kp) }\end{array}$} & \multirow{2}{*}{$\begin{array}{c}\text { Flecha al } \\
35 \%\left({ }^{*}\right) \\
\text { momento } \\
\text { de rotura } \\
(\mathrm{mm})\end{array}$} & \multicolumn{2}{|c|}{$\begin{array}{c}\text { Tensiones armadura } \\
\text { al } 35 \%\left(^{*}\right) \text { del momento } \\
\text { de rotura } \\
\left(\mathrm{kp} / \mathrm{cm}^{2}\right)\end{array}$} \\
\hline & & Inicio & $\begin{array}{l}\text { Ancho } \\
0,3 \mathrm{~mm}\end{array}$ & & $\begin{array}{l}\text { Armadura } \\
\text { original }\end{array}$ & $\begin{array}{c}\text { Armadura } \\
\text { refuerzo }\end{array}$ \\
\hline Original & $\begin{array}{l}1955 \\
1941 \\
1878\end{array}$ & $\begin{array}{l}761 \\
618 \\
761\end{array}$ & $\begin{array}{l}1334 \\
1478 \\
1621\end{array}$ & $\begin{array}{l}6,5 \\
5,2 \\
5,3\end{array}$ & $\begin{array}{l}1432 \\
1145 \\
1191\end{array}$ & $\begin{array}{l}- \\
- \\
-\end{array}$ \\
\hline V. medio (1) & 1925 & 713 & 1478 & 5,7 & 1256 & - \\
\hline $\begin{array}{l}\text { Refuerzo } \\
\text { malla y } \\
\text { mortero }\end{array}$ & $\begin{array}{l}3365 \\
3130 \\
3111\end{array}$ & $\begin{array}{l}556 \\
862 \\
862\end{array}$ & $\begin{array}{l}1722 \\
1435 \\
1579\end{array}$ & $\begin{array}{l}5,4 \\
5,9 \\
5,1\end{array}$ & $\begin{array}{l}1396 \\
1670 \\
1535\end{array}$ & $\begin{array}{l}2720 \\
2542 \\
2423\end{array}$ \\
\hline V. medio (2) & 3202 & 760 & 1579 & 5,5 & 1534 & 2562 \\
\hline$(2) /(1)$ & 1,66 & 1,06 & 1,06 & 0,96 & 1,22 & - \\
\hline $\begin{array}{l}\text { Ref. malla } \\
\text { y hormigón } \\
\text { proyectado }\end{array}$ & $\begin{array}{l}3253 \\
3230 \\
3230\end{array}$ & $\begin{array}{l}1443 \\
1586 \\
1443\end{array}$ & $\begin{array}{l}1586 \\
1872 \\
2159\end{array}$ & $\begin{array}{l}0,4 \\
0,5 \\
0,5\end{array}$ & $\begin{array}{r}142 \\
31 \\
25\end{array}$ & $\begin{array}{r}160 \\
59 \\
46\end{array}$ \\
\hline V. medio (3) & 3238 & 1491 & 1872 & 0,5 & 66 & 88 \\
\hline$(3) /(1)$ & 1,68 & 2,09 & 1,26 & 0,09 & 0,05 & - \\
\hline
\end{tabular}

( $^{*}$ ) Considerando que el valor del $35 \%$ de la carga de rotura en ensayo rápido puede suponer aproximadamente el valor del momento de servicio en la utilización del forjado. 


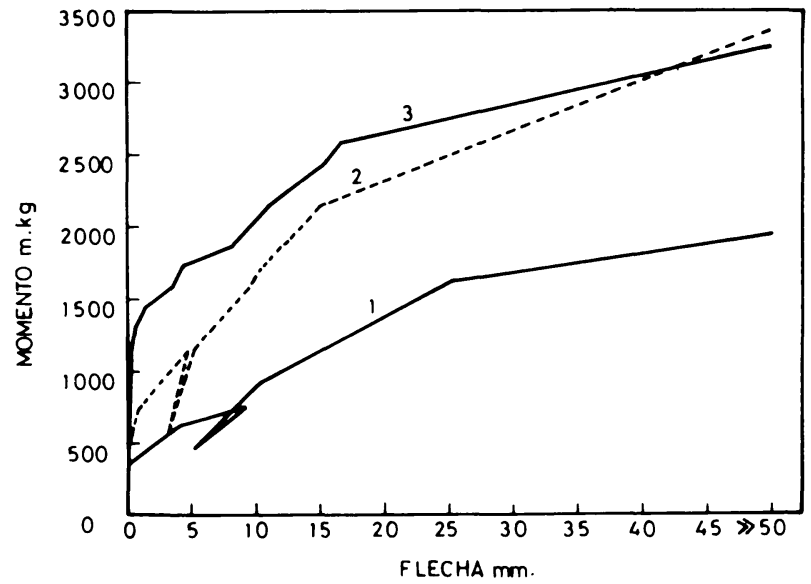

Gráfico 1.-Curvas momento en el centro de vano-deformación en cen tro de vano de los tres tipos de forjados ensayados.

1.-Forjado original, sin reforzar.

2.- Forjado reforzado con malla y mortero adherente.

3.- Forjado reforzado con malla y hormigón proyectado.

El valor de $\gg 50 \mathrm{~mm}$ en la fecha quiere indicar la correspondiente a la rotura. No se ha indicado en el gráfico para mantener buenas pro porciones en las pequeñas deformaciones.

\section{Forjados reforzados con malla y mortero adherente}

En todos los casos se produjo rotura a flexión en la zona de máximos momentos flectores con rotura a tracción de la armadura del mallazo recubierta con mortero adherente.

\section{Forjados reforzados con malla y hormigón proyectado}

La forma de rotura fue análoga al caso anterior. Conviene señalar, sin embargo, su forma peculiar de funcionamiento ya que durante un gran intervalo de la carga los forjados no presentaban en este caso fisuración apreciable, ni toma de tensión importante por las armaduras, hasta la formación, a una carga determinada, de una grieta bruscamente. Esto no indicaba la rotura de los forjados sino que pasaban a comportarse de forma totalmente análoga al caso anterior, y refleja una importante colaboración a tracción de la capa del hormigón proyectado.

\section{COEFICIENTES DE SEGURIDAD}

En el cuadro n. 3 se presentan globalmente el conjunto de cifras teóricas de rotura, los valores reales obtenidos y los factores de corrección comportamiento real/modelo teórico que permitirán sacar las conclusiones correspondientes.

\section{CONCLUSIONES}

Analizando todo lo expuesto anteriormente se puede hacer, para los refuerzos de las zonas de momento po-
CUADRO N. 3 COEFICIENTES DE SEGURIDAD: REFUERZOS EN FLEXION

\begin{tabular}{|c|c|c|c|c|}
\hline \multirow{2}{*}{$\begin{array}{l}\text { Tipo de } \\
\text { forjado }\end{array}$} & \multirow{2}{*}{$\begin{array}{l}\text { Forma } \\
\text { rotura }\end{array}$} & \multicolumn{2}{|c|}{$\begin{array}{l}\text { Esfuerzo de rotura (m.kp) } \\
\text { por vigueta }\end{array}$} & \multirow{2}{*}{$\begin{array}{c}\gamma_{n} \\
(2) /(1)\end{array}$} \\
\hline & & Calculado (1) & Real (2) & \\
\hline Original & Flexión & 1.830 & $\begin{array}{l}1.955 \\
1.941 \\
1.878\end{array}$ & $\begin{array}{l}1,07 \\
1,06 \\
1,03\end{array}$ \\
\hline $\begin{array}{l}\text { Ref. malla } \\
\text { mortero }\end{array}$ & Flexión & 3.031 & $\begin{array}{l}3.365 \\
3.130 \\
3.111 \\
\end{array}$ & $\begin{array}{l}1,11 \\
1,03 \\
1,03\end{array}$ \\
\hline $\begin{array}{l}\text { Ref. malla y } \\
\text { horm. proy. }\end{array}$ & Flexión & 3.031 & $\begin{array}{l}3.253 \\
3.230 \\
3.230\end{array}$ & $\begin{array}{l}1,07 \\
1,06 \\
1,07\end{array}$ \\
\hline
\end{tabular}

sitivo de forjados de semiviguetas de condiciones análogas a las de la experimentación realizada, las siguientes consideraciones:

- La teoría y supuestos del cálculo a flexión se han correspondido muy fielmente con los resultados obtenidos en las pruebas. La realidad supera ligeramente la evaluación teórica $\left(\gamma_{n}=1,07,1,06,1,03\right)$.

- Los refuerzos a flexión efectuados añadiendo una armadura en forma de malla inferior, sea colocada con mortero adherente, sea mediante hormigón proyectado, han funcionado de forma monolítica con el forjado original, presentando una gran regularidad de resultados y una relación de resistencias real/cálculo ligeramente mayor que en las piezas originales $\left(\gamma_{\mathrm{n}}=1,11,1,03,1,03,1,07,1,06,1,06\right)$.

- No era esperable teóricamente este resultado por el hecho de que la nueva armadura está adherida en un $85 \%$ a la parte inferior de las bovedillas de relleno y sólo en un $15 \%$ a la semivigueta resistente.

- En efecto, no habiendo armaduras de cosido entre el refuerzo añadido y el forjado original, el estado límite último de esfuerzo rasante está condicionado por el valor que pueda asignarse a dicho esfuerzo en el contacto entre el mortero adherente o el gunitado y la cara inferior de las semiviguetas resistentes. No existen en las normas criterios uniformes sobre el rasante de agotamiento proporcionado por el hormigón sólo. Con nuestros materiales puede oscilar desde resistencia nula, que preconiza alguna norma, hasta $6 \div 8 \mathrm{kp} / \mathrm{cm}^{2}$ en superficies intencionadamente rugosas que no es nuestro caso. En las condiciones de la experimentación quizás se podría contar con resistencias entre 0 y $3 \mathrm{kp} / \mathrm{cm}^{2}$.

El rasante que se ha producido para las cargas de rotura de los forjados reforzados, suponiendo transmisión a través sólo de las caras inferiores de las

http://informesdelaconstruccion.revistas.csic.es 
semiviguetas, $15 \%$ de la superficie total, y rotura de tipo frágil, tiene un valor de $4,4 \mathrm{kp} / \mathrm{cm}^{2}$ claramente superior al intervalo $0-3 \mathrm{kp} / \mathrm{cm}^{2}$ de resistencia última atribuible. Parece, pues, que puede admitirse una colaboración de las bovedillas de hormigón poroso en la transmisión de los esfuerzos rasantes, aun cuando no se cuente habitualmente con ellas en los cálculos.

- Debe considerarse sin embargo que la carga uniformemente repartida de normas produce más cortante, para igual momento, que las cuatro cargas transversales con que se han hecho los ensayos, descritas anteriormente. Si los ensayos se hubieran efectuado con carga uniformemente repartida el esfuerzo cortante máximo puntual en el apoyo puesto en juego hubiera sido el $33 \%$ superior al alcanzado en las pruebas, sin que se pueda afirmar si hubiera o no fallado por rasante la unión del refuerzo con el forjado original, aunque también debe considerarse que en los ensayos efectuados se produce en la proximidad de la primera carga bastante mayores cortante y momento que en la situación con carga uniformemente repartida.

- Estas últimas consideraciones y el no haberse observado ningún síntoma de fallo por rasante en la zona próxima a los apoyos durante los ensayos, habiéndose llegado, sin advertir ningún despegue, a la rotura de armaduras en el centro, hace confiar en la eficacia de adherir un mortero especial u hormigón proyectado con una armadura repartida uniformemente en la parte inferior de los forjados de semiviguetas, cuestión considerada muy problemática a nivel de planteamiento.

Como cada caso particular presenta diferentes relaciones flector/cortante, calidad de bovedillas, y otras peculiaridades, podría ser aconsejable la realización de algún ensayo después de diseñados casos reales de refuerzo de este tipo.

- La aplicación de la fórmula de Branson de cálculo aproximado de flechas a los forjados originales y su comparación con los valores experimentales nos lleva a observar que, para la carga de servicio, que coincide con el inicio de la fisuración, la flecha real supera en un $50 \%$ la flecha calculada y para cargas mayores, hasta rotura, se produce una buena coincidencia entre valor calculado y real.
- Desde el punto de vista de la deformación los forjados reforzados con malla adhesiva han presentado un comportamiento peculiar.

Los de malla sujeta con mortero adherente han mostrado en la realidad valores de flecha muy superiores a los calculados según la fórmula citada. Probablemente la aplicación del mortero con llana no rellena totalmente los huecos entre armadura de refuerzo y forjado, dando lugar a una deformabilidad mayor que la correspondiente a la resistencia a flexión que proporcionan las armaduras añadidas.

Por el contrario la adherencia de armaduras con hormigón proyectado embebe perfectamente las armaduras, sin dejar huecos, y crea una capa, la del gunitado, particularmente dura. Ello hace que las flechas, hasta que se produce la fisuración, son mucho más pequeñas que las reflejadas por las fórmulas. Una vez fisurado el refuerzo y el forjado, la fórmula de Branson queda ajustada a la realidad.

- Los dos métodos de refuerzo a flexión utilizados se prestan a campos de aplicación diversos. El hormigón proyectado es apto para su uso en estructuras abiertas, debido a la suciedad inherente del proceso y a la imprecisión de la proyección, siendo por otra parte su coste por $\mathrm{m}^{2}$ relativamente bajo. EI mortero adherente en cambio es útil para locales pequeños, en los que el factor coste del material no llega a ser importante, y sí la facilidad de ejecución.

\section{BIBLIOGRAFIA}

1. VIEITEZ, J. A. y RAMIREZ, J. L. (1985) Datos orientativos sobre la evolución de defectos en la construcción, en España. Hormigón y acero $n^{\circ} 157,101-110$

2. CEB 162 (1983) Asessment of concrete structures and design procedures for upgrading (redesign). Praga $288 \mathrm{p}$.

3. FIP (1985) Maintenance, repair and strengthening of existing structures. $84 \mathrm{p}$.

4. IABSE (1982) Strengthening of building structures. Diagnosis and therapy. Venezia.

5. FERNANDEZ CÁNOVAS, M. (1984) Patologia y terapéutica del hormigón armado. Dossat S. A. Madrid $620 \mathrm{p}$.

6. TASSIOS T. P. and VASSILIOU G. (1975) Mechanical behaviour of repaired R.C. structures. Colloquium Inter-Associations Behaviour in service of concrete structures. Liege Tome III, 1055-1069.

7. CALAVERA, J. (1986) Cálculo, construcción y patologia de forjados de edificación. INTEMAC. Madrid $670 \mathrm{p}$. 